Wert des $\log \mathrm{ft}$ für den $2,13 \mathrm{MeV} \beta$-Übergang ergibt

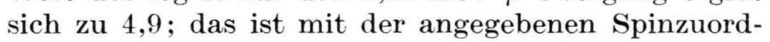
nung nicht unverträglich. Der nicht beobachtete Übergang zwischen den Grundzuständen sollte zweifach verboten sein. Die Gesamtzerfallsenergie von $2,45 \pm 0,03$ $\mathrm{MeV}$ paßt gut in das Jensen-Sueß-Diagramm ${ }^{13}$.

Nach Abschluß unserer Messungen wurde uns das Manuskript einer Arbeit von Jordan u. a. ${ }^{14}$ zugänglich. Das dort angegebene Zerfallsschema stimmt im wesentlichen mit unserem überein. Für die Höhe des

${ }_{13}$ O. Haxel, J. H. D. Jensen u. H. E. SueB, Ergebn. exakt. Naturwiss. 26, 244 [1952].

${ }_{14}$ W. C. Jordan, S. B. Burson u. J. M. LeBlanc, Phys. Rev., im Druck; kurze Zusammenfassung in zweiten angeregten Niveaus in $\mathrm{V}^{51}$ werden $0,92 \mathrm{MeV}$ angegeben, für die gesamte Zerfallsenergie des $\mathrm{Ti}^{51}$ $2,6 \pm 0,2 \mathrm{MeV}$.

Herrn Professor W. Bothe danken wir herzlich für sein förderndes Interesse, Herrn Professor J. H. D. Jensen für interessante Diskussionen und den Herren Dr. L. Koester und Dr. O. Knecht für die Durchführung der Bestrahlungen.

Für die Untersuchung wurden Apparate der Deutschen Forschungsgemeinschaft mitbenutzt.

Bull. Amer. Phys. Soc. 29, Nr. 7, S. 15 [1954]. Wir danken den Verfassern für die freundliche Übersendung ihres Manuskripts.

\section{Zur Abhängigkeit des Trenneffektes von der Gegenstromzirkulation in der Gaszentrifuge}

\author{
Von H. G. Hertz und E. Nann \\ Institut für Physikalische Chemie der Universität \\ Hamburg \\ (Z. Naturforschg. 10a, 170-171 [1955]; eingeg. am 10. Januar 1955)
}

In einer früheren Arbeit ${ }^{1}$ war über Isotopenanreicherungsversuche am Xenon berichtet worden, die bei kontinuierlicher Betriebsweise an einem an anderer Stelle beschriebenen Zentrifugengerät ${ }^{2} \mathrm{zu}$ erheblichen Verstärkungen des elementartheoretischen Trenneffektes geführt hatten. Verschiedene theoretische Untersuchungen $^{3,4,5}$ hatten zum Ziel, die durch Gegenstromzirkulation im Zentrifugenrotor entstehenden Trennverstärkungen zu berechnen. Die unter gewissen Vereinfachungen durchgeführte Rechnung von Martin und $\mathrm{Kuhn}^{4}$ macht eine numerische Voraussage des zu erwartenden Trennfaktors möglich.

Die vorliegenden experimentellen Untersuchungen liefern eine qualitative Bestätigung der von Martin und Kuhn gefundenen Resultate. Die Arbeitsweise glich der bereits früher ${ }^{1}$ beschriebenen: Aus einem ca. 100 Liter fassenden Vorratsbehälter strömte das Xenon durch den Zentrifugenrotor in der Weise, daß das aus dem Rotor austretende Gas denselben zur Hälfte am oberen Ende und zur anderen Hälfte am unteren Ende verließ. Die Isotopenverschiebung von $\mathrm{X}^{129}$ und $\mathrm{X}^{136}$ dieser beiden Stromhälften, $\left(n_{1} / n_{2}\right)_{L} /\left(n_{1} / n_{2}\right)_{0}$, wurde gemessen. Martin und $\mathrm{Kuhn}^{4}$ leiten ihre Formeln $\mathrm{ab}$ unter der Voraussetzung, daß die eine der beiden Komponenten der anderen in kleiner Menge beigemischt ist. Diese Voraussetzung ist bei den vorliegenden Versuchen nicht gut erfüllt. Unter Berücksichtigung dieser Tatsache kann man näherungsweise die Endformel für die Anreicherung in axialer Richtung an der Stelle $z$

${ }^{1}$ H. G. Hertz u. E. Nann, Z. Elektrochem. 58, 612 [1954].

${ }^{2}$ K. Beyerle, W. Groth, P. Harteck u. H. Jensen, Beihefte zur Angew. Chem. und Chem. Ing. Technik Nr. 59 (1950), Verlag Chemie Weinheim/Bergstraße. gegenüber der Stelle 0 schreiben $(z$-Richtung $=$ Richtung des inneren Axialstromes):

$$
\begin{gathered}
\frac{\left(\gamma_{2}\right)_{0}}{\left(\gamma_{2}\right)_{z}}=e^{\frac{2 \cdot \delta \cdot u}{u^{2} \tau_{\mathrm{I}}+2 D} \cdot \frac{1-\bar{\gamma}_{2}}{1-2 \bar{\gamma}_{2} \delta} \cdot z} \\
\delta=\frac{\pi^{2} v^{2}\left(M_{2}-M_{1}\right) r_{\mathrm{a}}^{2}}{R T}, \tau_{\mathrm{D}}=\frac{r_{\mathrm{a}}{ }^{2}}{2 D},
\end{gathered}
$$

wo $\gamma_{2}$ den Molenbruch der schweren Komponente, $\bar{\gamma}_{2}$ den Molenbruch des schweren im normalen Gas und $u$ die axiale Konvektionsgeschwindigkeit bedeutet. Variiert $u$ von 0 gegen sehr große Werte, so soll der Trennfaktor gemäß Gl. (1) von 1 kommend ein Maximum durchlaufen und wieder gegen 1 gehen. Das Maximum liegt bei der Geschwindigkeit $u_{0}=2 D / r_{\mathrm{a}}$. Dann liefert Gl. (1) auf die ganze Länge $L$ des Rotors bezogen

$$
\left\{\frac{\left(\gamma_{2}\right)_{0}}{\left(\gamma_{2}\right)_{L}}\right\}_{\max }=e^{\frac{\delta\left(1-\bar{\gamma}_{2}\right)}{r_{\mathrm{n}}\left(1-2 \bar{\gamma}_{2} \delta\right)} L}=1,51
$$

bzw. für das Verhältnis der Konzentrationen leicht/ schwer:

$$
\left\{\frac{\left(n_{1} / n_{2}\right)_{L}}{\left(n_{1} / n_{2}\right)_{0}}\right\}_{\max } \approx 1,71 .
$$

Die Zahlenwerte entsprechen den Daten des benutzten Zentrifugengerätes: $v=39600 \mathrm{Min}^{-1}, r_{\mathrm{a}}=6,7 \mathrm{~cm}, L=$ innere Länge des Rotors $=65 \mathrm{~cm}, M_{1}=129, M_{2}=136, \bar{\gamma}_{2}$ (bezogen auf das binäre Gemisch $\mathrm{X}^{136}, \mathrm{X}^{129}$ ) $=0,25$, $T=293^{\circ} \mathrm{K}$. Die Temperatur $T=293^{\circ}$ ist ein geschätzter Mittelwert aus den Temperaturen des oberen und unteren Temperierelements (siehe unten). Der gemessene Maximalwert war $\left(n_{1} / n_{2}\right)_{L} /\left(n_{1} / n_{2}\right)_{0}=1,64$ bzw. $\left(\gamma_{2}\right)_{0} /\left(\gamma_{2}\right)_{L}=1,455$. Hätte man $L=59 \mathrm{~cm}$, so stimmten experimenteller und berechneter Wert überein, dieses würde bedeuten, daß die wirksame Austauschlänge nur $59 \mathrm{~cm}$ beträgt. Liegt die zwischen Aufwärts- und Abwärtsstrom ruhende Schicht nicht bei $r_{\mathrm{m}}=r_{\mathrm{a}} / 2$,

${ }^{3}$ Bramley, Science 92, 427 [1940].

${ }^{4}$ H. Martin u. W. Kuhn, Z. phys. Chem. A 189, 219 [1940].

5 Siehe auch K. Cohen, The Theory of Isotopic Separation, Mac Graw Hill, New York 1951. 
sondern bei $r_{0}=r_{\mathrm{a}} / \sqrt{2}$ gleiche (Strömungsquerschnitte), so erhält man nach Martin und Kuhn

$$
\left\{\left.\frac{\left(\gamma_{2}\right)_{0}}{\left(\gamma_{2}\right)_{L}}\right|_{\max }=e^{\frac{\delta \cdot L}{r_{n}} \sqrt{2} \frac{1-\bar{\gamma}_{2}}{1-2 \bar{\gamma}_{2} \delta}}=1,79 ;\right.
$$

über die wirkliche Lage der ruhenden Schicht lassen sich bisher noch keine Aussagen machen.

Die Zeitdauer bis zum Erreichen des vollen Trennwertes wird zu $\tau_{0}=L^{2} / 2 D$ angegeben, wenn $u=u_{0} \mathrm{bzw}$. $1 / s^{2} \cdot L^{2} / 2 D$, wenn $u=s u_{0}$. Es wird angenommen ${ }^{6}$, daß die Gegenstromzirkulation durch einen axialen Temperaturgradienten $\Delta T / L$ angetrieben wird und daß $u$ prop. $\Delta T$ ist.

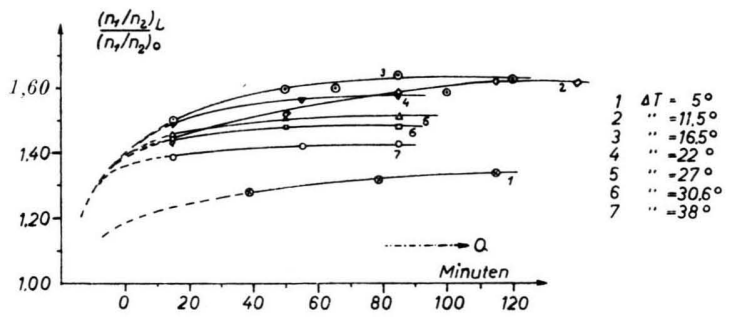

Abb. 1 zeigt den gemessenen Trenneffekt in Abhängigkeit von der Betriebszeit. Die Meßpunkte wurden an vier aufeinanderfolgenden Betriebstagen, an denen je zwei Versuche gemacht wurden, gewonnen. Zur Zeit $t \approx-20$ Min. wurde durch langsames Einströmen in den evakuierten Rotor mit der Füllung desselben begonnen, zur Zeit $t=-10$ Min. hatte die Füllung ihren Endwert erreicht $\left(p_{\text {Achse }}=60 \mathrm{~mm} \mathrm{Hg}\right)$, zur Zeit $t=0$ wurde durch Öffnen der Abzapfventile mit dem Gasdurchsatz begonnen. Dieser betrug ca. $10 \mathrm{ncm}^{3} /$ Min pro Zapfstrom, also $20 \mathrm{ncm}^{3} /$ Min. insgesamt. Die Kurven sind gekennzeichnet durch die zwischen den Temperierelementen durch Thermostaten eingestellte Temperaturdifferenz. Der genaue Temperaturverlauf im Rotor ist nicht bekannt. Das untere, kältere Temperierelement hatte eine Temperatur von

${ }^{6}$ H. Martin, Z. Elektrochem., 54, 120 [1950]. ca. $13^{\circ} \mathrm{C}$, der Sicherheitsmantel außen eine solche von ca. $17^{\circ} \mathrm{C}$, die leichte Fraktion trat unten aus.

Man erkennt bei kleiner Temperaturdifferenz $\left(5^{\circ}\right)$ ein langsames Ansteigen des Trenneffektes auf einen relativ niedrigen Wert, dann bei Erreichen der optimalen Temperaturdifferenz $\left(10-25^{\circ}\right)$ ein noch vergleichsweise langsames Ansteigen auf den Maximalwert. Bei weiterer Erhöhung der Temperaturdifferenz steigt der Trenneffekt schneller an, der Endwert liegt aber niedriger. Der Zeitbedarf für optimale Trennung ist $\mathrm{zu}$ ca. $100 \mathrm{Min}$. gefunden. Der theoretische Wert $\tau_{0}=L^{2} / 2 D$ gibt mit $D=1,2 \eta / \varrho$ an der Stelle $r=r_{\mathrm{a}} / 2$, $\eta=226$ Mikropoise bei $\vartheta=20^{\circ} \mathrm{C}: \tau_{0}=96 \mathrm{Min}$. in guter Úbereinstimmung. Der zu Abb. 1 gehörige Mengendurchsatz von ca. $1,2 \cdot 10^{-1} \mathrm{~g} /$ Min entspricht etwa dem unter Aufrechterhaltung optimaler Trennwirkung gerade noch zulässigen theoretisch angegebenen von $2 \cdot 1,2 \cdot \eta \pi r_{\mathrm{a}}{ }^{2} / L=7,2 \cdot 10^{-2} \mathrm{~g} / \mathrm{Min}$., wenn auch Hinweise dafür vorliegen, daß der Trenneffekt beim Mengendurchsatz 0 geringer ist, als bei dem oben angegebenen Durchsatz. Die Größe $Q$ der Abb. 1 stellt den elementartheoretischen Trennfaktor dar.

Bei weiteren Versuchen wurde der maximale Trennfaktor trotz der optimalen Temperatureinstellung nicht mehr erreicht $\left[\left(n_{1} / n_{2}\right)_{L} /\left(n_{1} / n_{2}\right)_{0}=1,56 \pm 0,03\right]$. Durch zusätzliche Kühlung des Öles am oberen Dämpflager bei Konstanthaltung von $\Delta T=17^{\circ}$ an den Temperierelementen fiel der Trenneffekt auf etwa 1,50, stieg aber durch Vergrößerung der Temperaturdifferenz an den Temperierelementen auf $27^{\circ}$ und Kühlung des Dämpflageröls wieder auf 1,60 an. Der Temperaturantrieb der Konvektion wird also nicht durch die Temperierelemente allein bestimmt, sondern resultiert aus der Wirkung der Temperierelemente und des am Sicherheitsmantel herabfließenden Öls. Gleichfalls konnte der Trenneffekt durch Abstellen des Wasserstoffstromes im Sicherheitsmantel verringert werden, das untere, kalte Ende des Rotors wurde dann nicht mehr genügend gekühlt.

Die Deutsche Forschungsgemeinschaft hat die vorliegende Arbeit durch Sachbeihilfen unterstützt, wofür wir ihr nochmals unseren Dank aussprechen.

\section{Zur Frage der Bindungsart in Carbiden von Übergangsmetallen}

Von H. Renner, G. Brauer und A. Faessler

Chemisches Laboratorium und Physikalisches Institut der Universität Freiburg i. Br.

(Z. Naturforschg. 10a, 171-172 [1955]; eingeg. am 15. Januar 1955)

Die Analyse der Röntgenemissionsspektren vermag in vielen Fällen Auskunft über den Bindungszustand eines bestimmten Atoms zu geben. Die Methode ist bisher vorwiegend auf Elemente niederer Ordnungszahl angewandt worden ${ }^{1}$; sie ist jedoch auch bei Elementen höherer Ordnungszahl von Erfolg, wenn man für die Untersuchung geeignete Übergänge wählt und Spektrographen nicht zu kleiner Dispersion anwendet.
Wir benutzten die Methode, um Auskunft über die Bindungsart des Niobs in den beiden Carbiden NbC und $\mathrm{Nb}_{2} \mathrm{C}$ zu erhalten. Als geeignet erschien uns die Linie $\mathrm{Nb} \mathrm{L} \beta_{2}\left(\mathrm{~L}_{\mathrm{III}}-\mathrm{N}_{\mathrm{IV}}, \mathrm{v}\right)$, die wegen der großen thermischen Stabilität der in Frage stehenden Verbindungen direkt mit Kathodenstrahlen erregt werden konnte. Die Linie ist verbreitert und besitzt für die verschiedenen Verbindungen eine charakteristisch verschiedene Form.

Wie schon $\mathrm{Haglund}{ }^{2}$ beobachtet hat, zeigt $\mathrm{L} \beta_{2}$ von Niobmetall ein Maximum bei $\lambda=5227,1 \mathrm{X}$ und die für

${ }^{1}$ Vgl. z. B.: A. Faessler u. M. Goehring, Naturwiss. 39, 169 [1952]; dort ausführliche Literaturangaben.

${ }^{2}$ Ph. Haglund, Diss. Uppsala 1941. 